\title{
Differences in Electromechanical Delay Subsequent to Neuromuscular Fatigue: A Potential Relationship to Physical Fitness Training
}

\section{Saeed El-Ashker*}

\author{
* Faculty of Physical Education, Mansoura University, Mansoura, Egypt
}

\section{Introduction}

One of the very important factors in combat sports success is lower body strength (Parr, 2018). when combat sport athletes (CSA) performing workouts, leg muscles occupied in all technical, tactical, and movements actions. Most workouts include exercises directed to develop leg strength (Ferruggia, 2008), punching skills (Oliver, Drinkell, \& Torborg, 2007), kicking actions (Palfrey \& Murch, 2013), and foot-work (Lissenden, 2015)

CSA need to perform unexpected accelerating and decelerating actions, quick changes of orientation, leaping and landing activities whether during training or competitions. These activities result in quick and unexpected actions reacts of the medial/lateral hamstrings which are required to steady the joints, besides neutralize the high load strength produced (McLean, Borotikar, \& Lucey, 2010; Smith et al., 2012). During these movements, several muscle endeavours take place with different co-contraction schemes needed to steady the joints.

It has been assumed that the reaction time is the much significant item connected with muscles and ligaments injuries (Hughes \& Watkins, 2006). Long reaction times might adversely influence the muscle's capability to rapidly steady the joints opposing the intensified external loads generated throughout athletic activities and then may rise risk of tears (Besier, Lloyd, \& Ackland, 2003; Blackburn, Riemann, Padua, \& Guskiewicz, 2004; McLean et al., 2010).

Electromechanical delay (EMD) is determined as the duration amongst the beginning of muscle movement and the beginning of power generation by that muscle movement (Zhou, Lawson, Morrison, \& Fairweather, 1995) with differ data values from 30 to a few-hundred ms (Shultz \& Perrin, 1999). Taking into account this time lapse and the necessity to set-up adequate muscular tension quickly to deliver dynamic joint constancy, EMD would be deemed when estimating muscular reactions (Shultz \& Perrin, 1999).

Neuromuscular fatigue significantly increases EMD (Howatson, 2010; Zhou, McKenna, Lawson, Morrison, \& Fairweather, 1996). As muscles assist to joint constancy, neuromuscular fatigue is 
recommended as a feature for non-contact injuries (Alentorn-Geli et al., 2009; Yu, Kirkendall, Taft, \& Garrett, 2002).

The aims of the existing study were: 1) to identify hamstrings neuromuscular output during functioning experimental trial in a fatigued and non-fatigued condition. 2) to examine the impacts of fatiguing on EMD of the knee flexors in combat sport athletes (CSA), healthy, and sedentary. 3) to explore the impacts of physical fitness condition on EMD in all groups.

\section{Methods}

Institutional review board (IRB) endorsement was attained. Study was conducted as a descriptive cross-section comparative study. Ninety male participants without any history of lower body injuries or any current complaint of the knee joint were incorporated in the study. Individuals were subdivided into three groups; Group $1=30$ combat sport athletes (Boxing=10; Kickboxing=7; Taekwondo=5; Karate=5; Judo=4), Group $2=30$ healthy individuals, and Group $3=30$ sedentary individuals.

Participants retained an informed consent to contribute in the study and got an information sheet to explain the empirical protocol of the study. A Health Questionnaire (PAR-Q) was utilized. Within 2 weeks prior to conducting the experiment tests, participants were invited to visit the sports medicine research laboratory in order to make sure that they are familiar with the laboratory and enlightened with the experimental procedures.

\section{Study protocols}

Testing protocols followed the laboratory techniques and instructions. Raw data was collected and set aside for analytical usage privately on both electronic and hardcopy, classifying and coding all participants' consequences to maintain confidentiality of data for each of the participants and study' information. Participants were informed not to devour any caffeine during and the day until their tests. Also, they were charged not to fulfil any exercises within the 48 hours prior to the test day.

\section{Electromyography measurements (pre-test)}

EMD Assessment was carried out using the isokinetic dynamometer (Biodex, New York, USA) besides electromyography (EMG) telemetry system (Delsys Myomonitor III, Massachusetts, USA). The dynamometer and EMG were contacted together via a trigger case (Delsys). We used 
this method to guarantee that the created data from both EMG besides dynamometer were totally aligned, then having the onset of EMG activity concerning the onset of torques output. Formerly and next to starting testing measures, both the dynamometer plus to the EMG apparatus were calibrated consistent with manufacturer's guidelines.

Applicants were fastened in a prone position on the Biodex and the pelvis inactively flexed at 10$20^{\circ}$ (De Ste Croix, ElNagar, Iga, James, \& Ayala, 2015; De Ste Croix, Priestley, Lloyd, \& Oliver, 2015). The axis of rotational movement of the Biodex lever was arrayed with the lateral epicondyle of the femur. The braking pad stuff was placed near 3-cm higher to the osseous prominence of the ankle "medial malleolus", the foot is in comfortable state. Adjustable strapping for the pelvic. Movement ranged from $0^{\circ}$ to $90^{\circ}$ (whereas $0^{\circ}=$ full knee extension; and $90^{\circ}$ is for knee flexion).

EMG was used to examine hamstring muscles through performing eccentric peak torque contractions. In addition, it was used to measure time delay between onsets of working activation and the force generation and gives an impression about mechanical processes which happens inside the muscles (Lacourpaille, Hug, \& Nordez, 2013). Delsys electrodes used to record the surface EMG signal. Electrodes were planted onto the central point of Biceps Femoris muscle, the Semitendinosus muscle, and the Semimembranosus muscle (Botter et al., 2011; Shultz \& Perrin, 1999). EMD was performed pre-and-post fatiguing protocol.

\section{Fatiguing protocol (post-test)}

Determining EMD of the hamstring muscles before and after fatiguing protocol was obtained by assessing EMG records. The aimed peak torque level was fixed at $60 \%$ of peak torques, according to previous investigations (Bigland-Ritchie, Furbush, \& Woods, 1986; Häkkinen \& Komi, 1983) to produce a temporary reduction in muscular execution seen as a disappointment to sustain a specific predictable force (Edwards, 1981) ensuring neuromuscular fatigue when executing intermittent sub maximal contractions. Five to ten minutes following the baseline contractions tests, each participant was implemented the nominated fatiguing protocol, which comprise of periodical intermittent contractions utilizing a 0.6 duty cycle, including $6 \mathrm{~s}$ contraction followed by 4 s relaxation at $60 \%$ of maximal voluntary contractions until fatigue (Conchola, Thompson, \& Smith, 2013; Vøllestad, 1997). Comparable to earlier studies processes (Bilodeau, Erb, Nichols, Joiner, \& Weeks, 2001; Vøllestad, 1997) fatiguing test was discontinued by investigator once 
applicant isn't able yet to obtain his aimed peak torque level, despite executing maximum exertion. Immediately, after completing Fatiguing protocol, EMG records were recorded. Verbal encouragement was given during the fatiguing protocol and also during eccentric contractions (Campenella, Mattacola, \& Kimura, 2000; Kimura, Gulick, \& Lukasiewicz III, 1999) .

\section{Statistical analysis}

Data was analysed using SPSS for Windows (version 21.0, SPSS, Inc., Chicago, IL, USA). Kolmogorov-Smirnov test has been used to checked for homogeneity and skewness of the distribution for the raw data sets. Descriptive data (means \pm standard deviations) were computed for all variables. For the independent variables of knee angular velocities (60, 180, and $300 \%$ s), hamstring muscles (BF, SM, ST, and Max 'maximum muscle contraction of the three muscles'), and physical condition (CSA, healthy, and sedentary). The main dependant variable is EMD. Repeated measures analysis of the variance (RMANOVA) was utilized to evaluate the main effect of time, speed and interaction between them on the main outcome (EMD). Significant main effects were additional evaluated by using Bonferroni-corrected post hoc t-tests. The significance level was determined to P-value $\leq 0.05$.

\section{Results}

There was no statistically significant difference amongst the three confined groups in mean age, however, significant difference was observed in stature $(\mathrm{P}=<0.001)$ and body mass $(\mathrm{BM})(\mathrm{P}<$ 0.001) (see Table 1). In all three studied groups, the mean EMD was consistently increasing after increasing the speed (see Table 2, and figure 1).

Table 1: Demographic characteristics of participants

\begin{tabular}{ccccc}
\hline & $\begin{array}{c}\text { Combat sport athletes } \\
(\mathbf{N = 3 0})\end{array}$ & $\begin{array}{c}\text { Healthy } \\
(\mathbf{N}=30)\end{array}$ & $\begin{array}{c}\text { Sedentary } \\
(\mathbf{N = 3 0 )}\end{array}$ & P-value \\
\hline Age (years) & $22.35 \pm 3.25$ & $23.45 \pm 4.36$ & $22.94 \pm 3.74$ & 0.22 \\
Stature (m) & $1.77 \pm 0.04$ & $1.74 \pm 0.05$ & $1.72 \pm 0.04$ & $<0.01$ \\
BM $(\mathbf{K g})$ & $74.36 \pm 4.30$ & $79.52 \pm 6.88$ & $89.39 \pm 5.74$ & $<0.01$ \\
\hline
\end{tabular}


A significant main effect for both time and speed was observed across all muscle groups. Also, RNANOVA test revealed a significant interaction effect between time and speed in all muscle groups (see table 4, and figure 2). During maximum muscle contraction, the main effect for time, speed and time $\mathrm{x}$ speed were all significant $\left(\mathrm{F}_{1,74}=537.754, \mathrm{P}=<0.001-\mathrm{F}_{2,73}=770.646, \mathrm{P}=\right.$ $<0.001-\mathrm{F}_{2,73}=7.424, \mathrm{P}=<0.001$ respectively). Moreover, post-hoc analysis revealed that irrespective muscle, speed or group, mean EMD was significantly higher in post-fatigue compared to pre-fatigue [pre $=60.90 \mathrm{~ms}$, post $=42.49 \mathrm{~ms}, \mathrm{P}$ value $=<0.001,(43.32 \%$ increase $)]$.

Table 2: EMD Pre fatigue by group condition, muscle, and movement speed

\begin{tabular}{ccccc}
\hline & \multicolumn{3}{c}{ EMD Pre-Fatigue } & \\
\cline { 2 - 4 } & $\begin{array}{c}\text { CSA } \\
(\mathbf{N = 3 0})\end{array}$ & $\begin{array}{c}\text { Healthy } \\
(\mathbf{N = 3 0})\end{array}$ & $\begin{array}{c}\text { Sedentary } \\
(\mathbf{N}=\mathbf{3 0})\end{array}$ & combined \\
\hline Biceps femoris & $23.52 \pm 3.37$ & $25.64 \pm 4.11$ & $26.24 \pm 4.09$ & $25.13 \pm 3.99$ \\
\hline $60 \% / \mathrm{s}$ & $38.04 \pm 12.38$ & $41.08 \pm 10.51$ & $48.24 \pm 13.67$ & $42.45 \pm 12.83$ \\
$180 \% \mathrm{~s}$ & $51.00 \pm 7.26$ & $52.20 \pm 9.26$ & $53.60 \pm 8.699$ & $52.27 \pm 8.40$ \\
$300 \% \mathrm{~s}$ & & & & \\
Semimembranosus & $24.16 \pm 3.45$ & $26.56 \pm 4.20$ & $26.04 \pm 4.05$ & $25.59 \pm 3.99$ \\
$60 \% \mathrm{~s}$ & $38.52 \pm 11.00$ & $40.04 \pm 9.82$ & $47.36 \pm 13.81$ & $44.08 \pm 12.49$ \\
$180 \% \mathrm{~s}$ & $53.68 \pm 9.95$ & $53.92 \pm 11.55$ & $55.76 \pm 10.07$ & $54.45 \pm 10.45$ \\
$300 \% \mathrm{~s}$ & & & & \\
Semitendinosus & $24.52 \pm 4.05$ & $27.40 \pm 4.29$ & $27.08 \pm 4.08$ & $26.33 \pm 4.29$ \\
$60 \% \mathrm{~s}$ & $41.56 \pm 13.57$ & $43.96 \pm 11.63$ & $46.72 \pm 12.12$ & $44.08 \pm 12.48$ \\
$180 \% \mathrm{~s}$ & $58.68 \pm 8.17$ & $57.92 \pm 9.91$ & $57.96 \pm 8.09$ & $58.19 \pm 8.65$ \\
$300 \% \mathrm{~s}$ & & & & \\
$\mathbf{M a x}$ & $26.64 \pm 3.56$ & $28.92 \pm 3.72$ & $28.52 \pm 3.70$ & $28.03 \pm 3.75$ \\
$60 \% \mathrm{~s}$ & $46.80 \pm 11.48$ & $47.52 \pm 10.11$ & $55.28 \pm 10.70$ & $49.87 \pm 11.31$ \\
$180 \% \mathrm{~s}$ & $61.44 \pm 7.57$ & $61.92 \pm 7.90$ & $61.04 \pm 7.16$ & $61.47 \pm 7.48$ \\
$300 \% \mathrm{~s}$ & & & & \\
\hline
\end{tabular}

Figure 1: Maximum EMD Pre-fatiguing chart, classified by group condition, muscle, and movement speed 


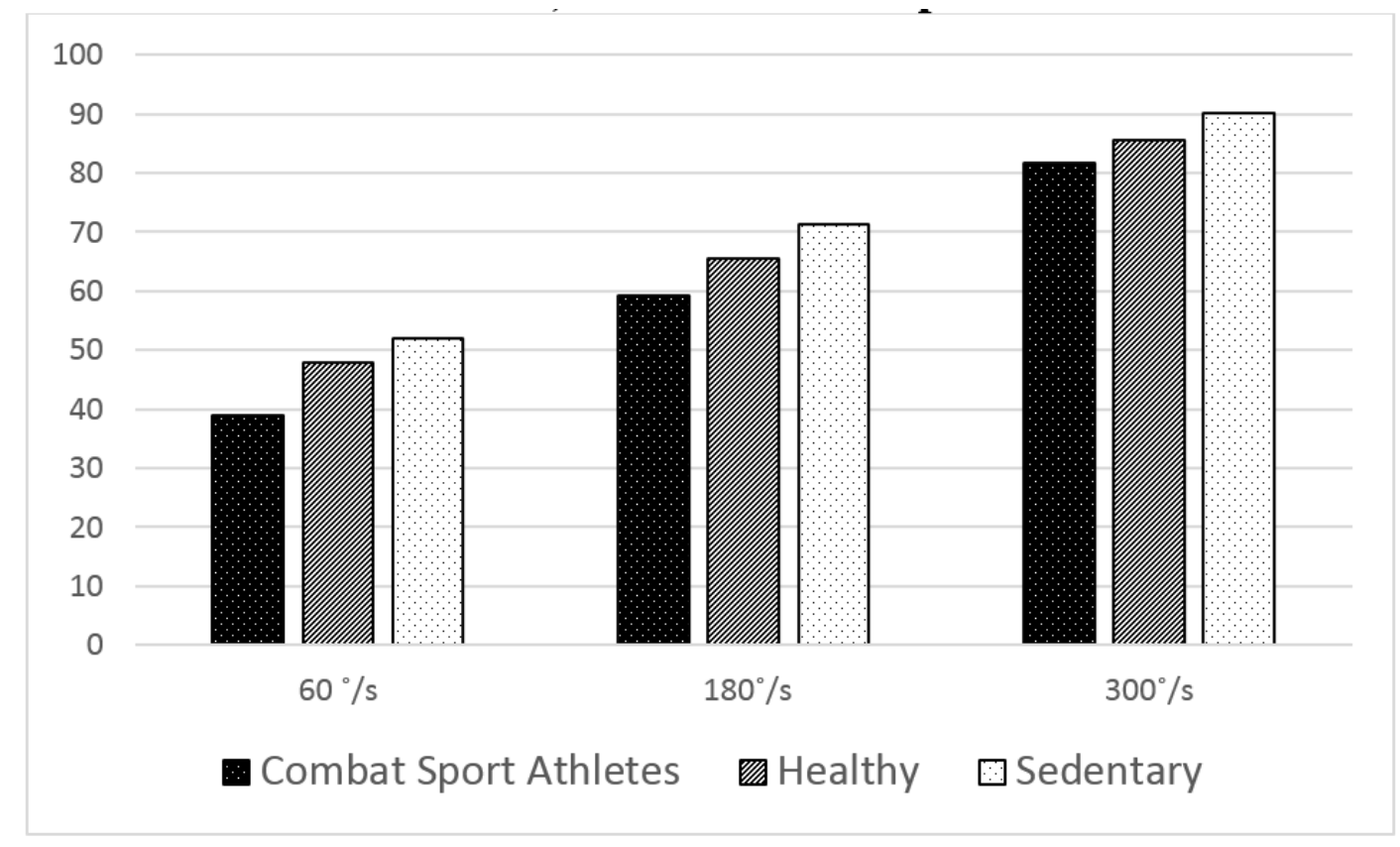

Table 3: EMD Post fatigue by group condition, muscle, and movement speed

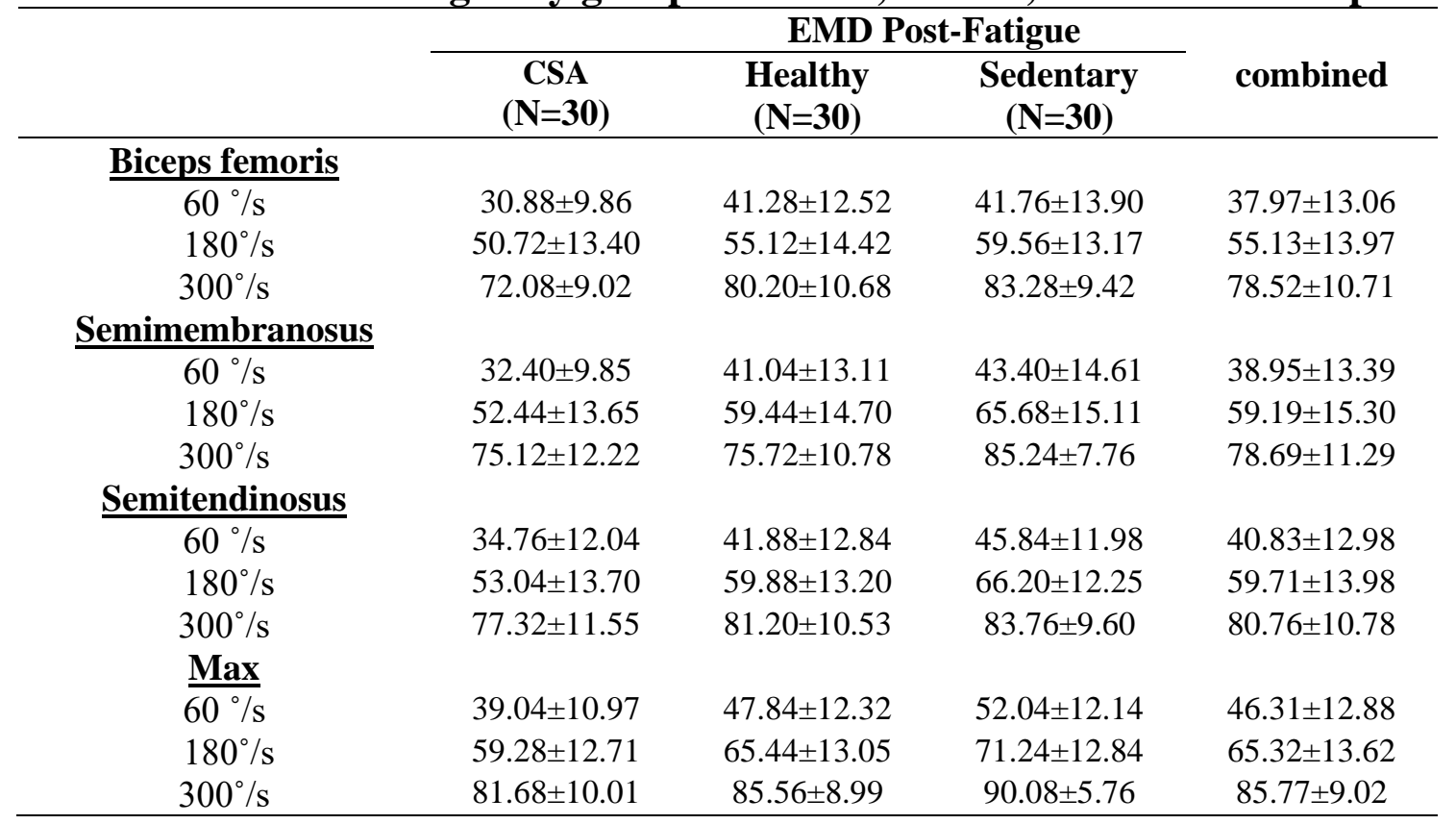

Figure 1: Maximum EMD Pre-fatiguing chart, classified by group condition, muscle, and movement speed 


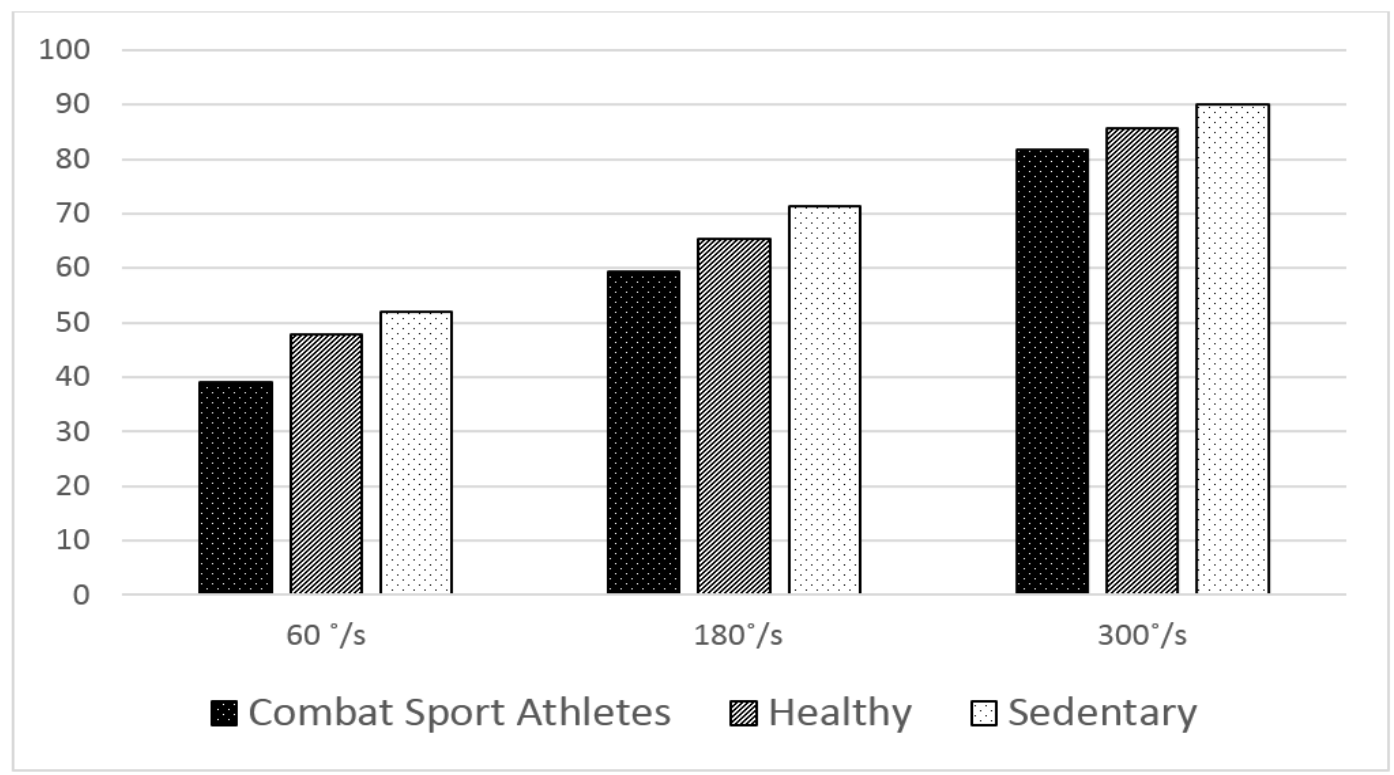

Irrespective of muscle, speed or time, mean EMD was higher in the sedentary group compared with the CSA group $[\mathrm{P}=<0.001,55.13 \pm 22.9 \mathrm{~ms}$ vs $47.68 \pm 19.9 \mathrm{~ms}$ (15.6\% increase)]. Furthermore, irrespective of muscle, speed or time, mean EMD was higher in the sedentary group compared with the healthy group $(\mathrm{P}=0.011,55.13 \pm 22.9 \mathrm{~ms}$ vs $51.72 \pm 19.9 \mathrm{~ms}$ [6.6\% increase $)$ ] (see Fig. 1).

Table 4: comparisons between different effects and different muscles

\begin{tabular}{lllll}
\hline Main effect & Muscle & df & F & P value \\
\hline Time & BM & 1 & 275.39 & $<0.001$ \\
& SM & 1 & 255.440 & $<0.001$ \\
& ST & 1 & 386.869 & $<0.001$ \\
Speed & MAX & 1 & 537.754 & $<0.001$ \\
& BM & 2 & 490.288 & $<0.001$ \\
& SM & 2 & 448.564 & $<0.001$ \\
\multirow{5}{*}{ Time x Speed } & ST & 2 & 575.251 & $<0.001$ \\
& MAX & 2 & 770.646 & $<0.001$ \\
& BM & 2 & 20.903 & $<0.001$ \\
& SM & 2 & 9.864 & $<0.001$ \\
& ST & 2 & 4.802 & 0.010 \\
& MAX & 2 & 7.424 & 0.001 \\
\hline
\end{tabular}

Figure 3: Effect of time (pre-post- fatiguing) on EMD, separated by group

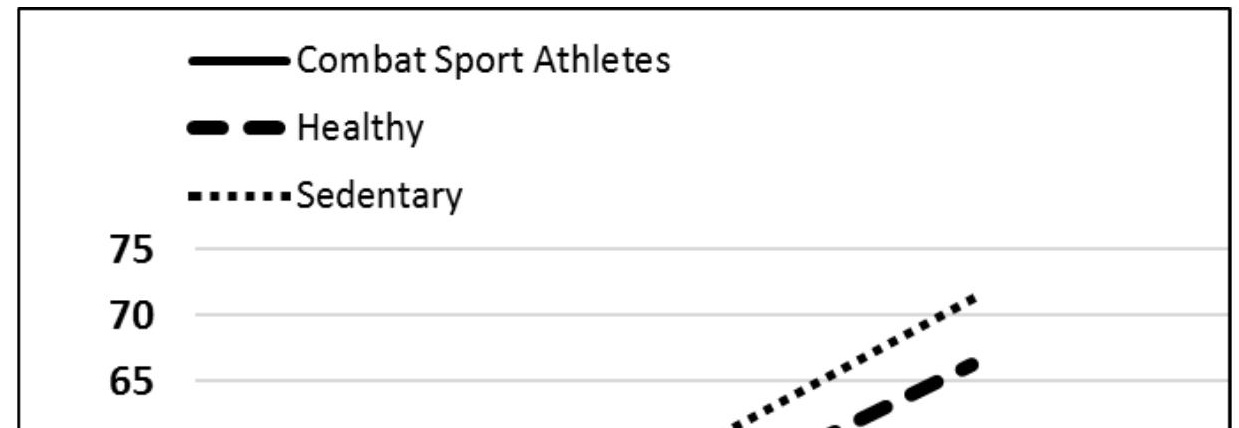


When comparing the three groups, a significant increase in EMD was observed in all muscles after taking time as main effect (see Table 4, and figure 3). The group comparison separated by each muscle was significant in all of comparisons except in comparison between healthy and sedentary groups in: $\mathrm{BM}(\mathrm{P}=0.146), \mathrm{SM}(\mathrm{P}=0.062), \mathrm{ST}(\mathrm{P}=0.160)$.

\section{Discussion:}

The most noteworthy finding in the current study is that neuromuscular fatigue caused by executing intermittent sub maximal contractions significantly increases the EMD, compromising neuromuscular control necessary to fix the joint in all studied groups (CSA, healthy and sedentary). These results were considerably higher in sedentary group relative to other physically good condition groups (CSA and healthy). A significant main effect between pre and post fatigue has been found, showing that the EMD was higher following the fatigue task $(\mathrm{P}<0.001)$, regardless of group, muscle, or contraction speed. It is hypothesized that this increase of EMD post-fatigue, which is recurrently stated in previous articles (Conchola et al., 2013; De Ste Croix, ElNagar, et al., 2015; De Ste Croix, Priestley, et al., 2015; Howatson, 2010; Mercer, Gleeson, Claridge, \& Clement, 1998; Zhou et al., 1996), is owing to metabolic inhibition of the muscle contractility and excitation-contraction coupling failure (Allen, Westerblad, Lee, \& Lannergren, 1992; Kent-Braun, 1999). This disadvantageous impact of fatigue as well as alteration in neuromuscular performance might lead to augment risk factors for knee injuries. 
To our knowledge, our study is the initial study to inspect the impact of fatigue on EMD throughout eccentric contractions of the hamstring muscles based on physical fitness conditions (e.g. CSA, healthy, and sedentary) in males. Previous studies have examined the impact of fatigue on EMD however within athlete populations without comparing with other different physical fitness conditions to generalize the benefit of the results. The results of the current study add and identify a new risk factor that affect EMD (e.g. physical fitness condition) beside to previous factors (e.g. type of muscle action (Cavanagh \& Komi, 1979); joint angle (De Ste Croix, ElNagar, et al., 2015; De Ste Croix, Priestley, et al., 2015; Mercer et al., 1998; Minshull, Rees, \& Gleeson, 2011); fatigue (Conchola, Thiele, Palmer, Smith, \& Thompson, 2015; De Ste Croix, ElNagar, et al., 2015; De Ste Croix, Priestley, et al., 2015; Mercer et al., 1998; Thompson, Conchola, \& Stock, 2015); the age (Conchola et al., 2013; De Ste Croix, Priestley, et al., 2015; Kim et al., 2011); and sex of the participants (De Ste Croix, ElNagar, et al., 2015; Kim et al., 2011; Shultz \& Perrin, 1999; Zhou et al., 1995).

The increment of EMD is alike to the results of earlier studies which presented fatigue inducing multiplies in EMD for hamstrings (Conchola et al., 2013). As EMD changes according to both the muscle-tendon unit structure (Minshull, Eston, Rees, \& Gleeson, 2012; Zhou et al., 1996) as well as muscle membrane action potential (Green, 1997; Zhou et al., 1996). Subsequently, inducing fatigue amplifies in EMD noticed in the current study might be a result of changes or deteriorations in these physiological mechanisms. In this regard, previous studies showed that a fatigue protocol of the hamstrings deducted significant reduces in stiffness of the muscle tendon unit and (Toumi et al., 2006), because stiffness characteristics of the muscle tendon unit affect the rate at which forces are sent out to the skeletal structures (Palmer, Jenkins, Thompson, Smith, \& Cramer, 2014; Watsford, Ditroilo, Fernandez-Pena, D'Amen, \& Lucertini, 2010) it is probable that declines in stiffness because of fatigue might augment EMD response time needed for transmission of forces throughout contractions/movements (Conchola et al., 2015).

The current study established that angular velocity has a significant effect and enlarged the EMD in the three examined muscles (Biceps femoris, Semimembranosus, Semitendinosus) with raising the angular velocity 'regardless of physical conditions. These propose that raising knee angular velocity might affect neuromuscular function of the hamstrings, causing a reduction and limit of knee joint constancy, and eventually providing to injury occurrence. On the other hand, few studies have inspected the effect of angular velocity on EMD (De Ste Croix, Priestley, et al., 2015). 
Other main result of the current study is the tendency for lesser EMD in the CSA participants compared with healthy and sedentary participants, within the pre and post-tests, along with all angular velocities. Findings are consistent with earlier studies inspected EMD of hamstring muscles through eccentric actions which found lower EMD in the power-trained boys compared with endurance-trained and non-athletes (Mitchell et al., 2011). These finding also emphasizes that physical training lead to advanced stiffness of the musculotendinous complex in males, and this result is in harmony with earlier investigations which demonstrated that greater hamstrings stiffness was linked with lower ACL load and injury risk, as musculotendinous stiffness is a adaptable feature; consequently drills and training which develop hamstring muscle stiffness might be significant enlargements to ACL injury prevention programs (Blackburn, Norcross, Cannon, \& Zinder, 2013). Therefore, it is possible that the physical training was prosperous in athletes and healthy than sedentary because of their intrinsic ability to create wellorganized muscle contraction. Consequently, the current study proposes that the elevated rate of lower EMD were induced by physical condition.

ACL injury is a usual and possible traumatic related injury, presenting with considerable morbidities, and the current study is seeking the risk factors affecting ACL injuries occurrence in the Kingdom of Saudi Arabia society. The current study designated that fatiguing compromises with neuromuscular control that affect the knee stabilization and these impacts are health specific with neuromuscular function reduction. The data highlighted that fatiguing would be discussed as an issue when recommending injury screening strategies in males. Similarly, results emphasise that intervention training programs should be implemented, and emphasis on neuromuscular fatigue strength, to improve neuromuscular response mechanisms.

\section{References}

Alentorn-Geli, E., Myer, G. D., Silvers, H. J., Samitier, G., Romero, D., Lazaro-Haro, C., \& Cugat, R. (2009). Prevention of non-contact anterior cruciate ligament injuries in soccer players. Part 1: Mechanisms of injury and underlying risk factors. Knee Surg Sports Traumatol Arthrosc, 17(7), 705-729. doi: 10.1007/s00167-009-0813-1

Allen, D. G., Westerblad, H., Lee, J. A., \& Lannergren, J. (1992). Role of excitation-contraction coupling in muscle fatigue. Sports Medicine, 13(2), 116-126.

Besier, T. F., Lloyd, D. G., \& Ackland, T. R. (2003). Muscle activation strategies at the knee during running and cutting maneuvers. Med Sci Sports Exerc, 35(1), 119-127. doi: 10.1249/01.mss.0000043608.79537.ab 
Bigland-Ritchie, B., Furbush, F., \& Woods, J. (1986). Fatigue of intermittent submaximal voluntary contractions: central and peripheral factors. J Appl Physiol, 61(2), 421-429.

Bilodeau, M., Erb, M. D., Nichols, J. M., Joiner, K. L., \& Weeks, J. B. (2001). Fatigue of elbow flexor muscles in younger and older adults. Muscle Nerve, 24(1), 98-106.

Blackburn, J. T., Norcross, M. F., Cannon, L. N., \& Zinder, S. M. (2013). Hamstrings stiffness and landing biomechanics linked to anterior cruciate ligament loading. J Athl Train, 48(6), 764-772. doi: 10.4085/1062-6050-48.4.01

Blackburn, J. T., Riemann, B. L., Padua, D. A., \& Guskiewicz, K. M. (2004). Sex comparison of extensibility, passive, and active stiffness of the knee flexors. Clin Biomech (Bristol, Avon), 19(1), 36-43.

Botter, A., Oprandi, G., Lanfranco, F., Allasia, S., Maffiuletti, N. A., \& Minetto, M. A. (2011). Atlas of the muscle motor points for the lower limb: implications for electrical stimulation procedures and electrode positioning. Eur J Appl Physiol, 111(10), 2461-2471. doi: 10.1007/s00421-011-2093-y

Campenella, B., Mattacola, C. G., \& Kimura, I. F. (2000). Effect of visual feedback and verbal encouragement on concentric quadriceps and hamstrings peak torque of males and females. Isokinetics and Exercise Science, 8(1), 1-6.

Cavanagh, P. R., \& Komi, P. V. (1979). Electromechanical delay in human skeletal muscle under concentric and eccentric contractions. Eur J Appl Physiol Occup Physiol, 42(3), 159-163.

Conchola, E. C., Thiele, R. M., Palmer, T. B., Smith, D. B., \& Thompson, B. J. (2015). Effects of neuromuscular fatigue on electromechanical delay of the leg extensors and flexors in young men and women. Muscle Nerve, 52(5), 844-851. doi: 10.1002/mus.24598

Conchola, E. C., Thompson, B., \& Smith, D. (2013). Effects of neuromuscular fatigue on the electromechanical delay of the leg extensors and flexors in young and old men. Eur J Appl Physiol, 113(9), 2391-2399.

De Ste Croix, M. B., ElNagar, Y. O., Iga, J., James, D., \& Ayala, F. (2015). Electromechanical delay of the hamstrings during eccentric muscle actions in males and females: Implications for non-contact ACL injuries. $J$ Electromyogr Kinesiol, 25(6), 901-906. doi: 10.1016/j.jelekin.2015.09.006

De Ste Croix, M. B., Priestley, A. M., Lloyd, R. S., \& Oliver, J. L. (2015). ACL injury risk in elite female youth soccer: Changes in neuromuscular control of the knee following soccerspecific fatigue. Scand J Med Sci Sports, 25(5), e531-538. doi: 10.1111/sms.12355

Edwards, R. H. (1981). Human muscle function and fatigue. Ciba Found Symp, 82, 1-18.

Ferruggia, J. (2008). Fit to Fight: An Insanely Effective Strength and Conditioning Program for the Ultimate MMA Warrior: Penguin Publishing Group.

Green, H. J. (1997). Mechanisms of muscle fatigue in intense exercise. J Sports Sci, 15(3), 247256. doi: 10.1080/026404197367254

Häkkinen, K., \& Komi, P. V. (1983). Electromyographic and mechanical characteristics of human skeletal muscle during fatigue under voluntary and reflex conditions. Electroencephalogr Clin Neurophysiol, 55(4), 436-444.

Howatson, G. (2010). The impact of damaging exercise on electromechanical delay in biceps brachii. J Electromyogr Kinesiol, 20(3), 477-481. doi: 10.1016/j.jelekin.2009.08.002

Hughes, G., \& Watkins, J. (2006). A risk-factor model for anterior cruciate ligament injury. Sports Medicine, 36(5), 411-428. 
Kent-Braun, J. A. (1999). Central and peripheral contributions to muscle fatigue in humans during sustained maximal effort. Eur J Appl Physiol Occup Physiol, 80(1), 57-63. doi: $10.1007 / \mathrm{s} 004210050558$

Kim, J. W., Kwon, Y., Chung, H. Y., Eom, G. M., Jun, J. H., Chung, J. S., \& Park, B. K. (2011). Age-sex differences in the hip abductor muscle properties. Geriatr Gerontol Int, 11(3), 333-340.

Kimura, I. F., Gulick, D. T., \& Lukasiewicz III, W. C. (1999). Effect of visual feedback and verbal encouragement on eccentric quadriceps and hamstrings peak torque. Res Sports Med, 9(1), 61-70.

Lacourpaille, L., Hug, F., \& Nordez, A. (2013). Influence of passive muscle tension on electromechanical delay in humans. PloS one, 8(1), e53159.

Lissenden, H. (2015). The Complete Guide to Boxing Fitness: A non-contact boxing training manual: Bloomsbury Publishing.

McLean, S. G., Borotikar, B., \& Lucey, S. M. (2010). Lower limb muscle pre-motor time measures during a choice reaction task associate with knee abduction loads during dynamic single leg landings. Clin Biomech (Bristol, Avon), 25(6), 563-569. doi: 10.1016/j.clinbiomech.2010.02.013

Mercer, T. H., Gleeson, N. P., Claridge, S., \& Clement, S. (1998). Prolonged intermittent high intensity exercise impairs neuromuscular performance of the knee flexors. Eur J Appl Physiol Occup Physiol, 77(6), 560-562. doi: 10.1007/s004210050377

Minshull, C., Eston, R., Rees, D., \& Gleeson, N. (2012). Knee joint neuromuscular activation performance during muscle damage and superimposed fatigue. J Sports Sci, 30(10), 10151024. doi: 10.1080/02640414.2012.682084

Minshull, C., Rees, D., \& Gleeson, N. P. (2011). Joint angle affects volitional and magneticallyevoked neuromuscular performance differentially. J Electromyogr Kinesiol, 21(4), 672677. doi: 10.1016/j.jelekin.2011.03.008

Mitchell, C., Cohen, R., Dotan, R., Gabriel, D., Klentrou, P., \& Falk, B. (2011). Rate of muscle activation in power-and endurance-trained boys. Int J Sports Physiol Perform, 6(1), 94105.

Oliver, I., Drinkell, P., \& Torborg, A. (2007). Boxing Fitness: Snowbooks Limited.

Palfrey, M., \& Murch, W. (2013). Sandbag Training for MMA and Combat Sports: CreateSpace Independent Publishing Platform.

Palmer, T. B., Jenkins, N. D., Thompson, B. J., Smith, D. B., \& Cramer, J. T. (2014). The relationship between passive stiffness and muscle power output: influence of muscle crosssectional area normalization. Muscle Nerve, 49(1), 69-75. doi: 10.1002/mus.23861

Parr, D. Y. (2018). Strength and Conditioning for Combat Sports. Marlborough; United Kingdom: Crowood.

Shultz, S. J., \& Perrin, D. H. (1999). Using surface electromyography to assess sex differences in neuromuscular response characteristics. J Athl Train, 34(2), 165-176.

Smith, H. C., Vacek, P., Johnson, R. J., Slauterbeck, J. R., Hashemi, J., Shultz, S., \& Beynnon, B. D. (2012). Risk factors for anterior cruciate ligament injury: a review of the literature - part 1: neuromuscular and anatomic risk. Sports Health, 4(1), 69-78. doi: $10.1177 / 1941738111428281$

Thompson, B. J., Conchola, E. C., \& Stock, M. S. (2015). Effects of age and muscle action type on acute strength and power recovery following fatigue of the leg flexors. Age, 37(6), 111. 
Toumi, H., Poumarat, G., Best, T. M., Martin, A., Fairclough, J., \& Benjamin, M. (2006). Fatigue and muscle-tendon stiffness after stretch-shortening cycle and isometric exercise. Appl Physiol Nutr Metab, 31(5), 565-572. doi: 10.1139/h06-034

Vøllestad, N. K. (1997). Measurement of human muscle fatigue. J Neurosci Methods, 74(2), 219227.

Watsford, M., Ditroilo, M., Fernandez-Pena, E., D'Amen, G., \& Lucertini, F. (2010). Muscle stiffness and rate of torque development during sprint cycling. Med Sci Sports Exerc, 42(7), 1324-1332. doi: 10.1249/MSS.0b013e3181ce509d

Yu, B., Kirkendall, D. T., Taft, T. N., \& Garrett, W. E., Jr. (2002). Lower extremity motor controlrelated and other risk factors for noncontact anterior cruciate ligament injuries. Instr Course Lect, 51, 315-324.

Zhou, S., Lawson, D. L., Morrison, W. E., \& Fairweather, I. (1995). Electromechanical delay in isometric muscle contractions evoked by voluntary, reflex and electrical stimulation. Eur J Appl Physiol Occup Physiol, 70(2), 138-145.

Zhou, S., McKenna, M. J., Lawson, D. L., Morrison, W. E., \& Fairweather, I. (1996). Effects of fatigue and sprint training on electromechanical delay of knee extensor muscles. Eur J Appl Physiol Occup Physiol, 72(5-6), 410-416. 